\title{
In vivo imaging reveals reduced activity of neuronal circuits in a mouse tauopathy model
}

\author{
Petar Marinković, ${ }^{1,2,3}$ Sonja Blumenstock, ${ }^{1,2,3}$ Pieter M. Goltstein, ${ }^{4}$ Viktoria Korzhova, ${ }^{1,2}$ \\ Finn Peters, ${ }^{1,2,3}$ Andreas Knebl',3 and Jochen Herms ${ }^{1,2,3}$
}

See Busche (doi:10.1093/brain/awz060) for a scientific commentary on this article.

Pathological alterations of tau protein play a significant role in the emergence and progression of neurodegenerative disorders. Tauopathies are characterized by detachment of the tau protein from neuronal microtubules, and its subsequent aberrant hyperphosphorylation, aggregation and cellular distribution. The exact nature of tau protein species causing neuronal malfunction and degeneration is still unknown. In the present study, we used mice transgenic for human tau with the frontotemporal dementia with parkinsonism-associated P301S mutation. These mice are prone to develop fibrillar tau inclusions, especially in the spinal cord and brainstem. At the same time, cortical neurons are not as strongly affected by fibrillar tau forms, but rather by soluble tau forms. We took advantage of the possibility to induce formation of neurofibrillary tangles in a subset of these cortical neurons by local injection of preformed synthetic tau fibrils. By using chronic in vivo two-photon calcium imaging in awake mice, we were able for the first time to follow the activity of individual tangle-bearing neurons and compare it to the activity of tangle-free neurons over the disease course. Our results revealed strong reduction of calcium transient frequency in layer $2 / 3$ cortical neurons of P301S mice, independent of neurofibrillary tangle presence. These results clearly point to the impairing role of soluble, mutated tau protein species present in the majority of the neurons investigated in this study.

1 Department for Translational Brain Research, German Center for Neurodegenerative Diseases (DZNE), Munich, Germany

2 Munich Cluster of Systems Neurology (SyNergy), Ludwig-Maximilians-University Munich, Munich, Germany

3 Center for Neuropathology and Prion Research, Ludwig-Maximilians-University Munich, Munich, Germany

4 Max Planck Institute of Neurobiology, Martinsried, Germany

Correspondence to: Prof. Jochen Herms

Feodor-Lynen-Straße 17, 81377, Munich, Germany

E-mail: jochen.herms@med.uni-muenchen.de

Keywords: tau; neurofibrillary tangles; two-photon imaging; seeding; P301S mice

Abbreviations: FSB $=$ [(trans,trans)-1-fluoro-2,5-bis(3-hydroxycarbonyl-4-hydroxy)styrylbenzene $]$; FDP-17 = frontotemporal dementia with parkinsonism linked to chromosome 17 ; NFT = neurofibrillary tangle; tau-PFF = tau preformed fibril

\section{Introduction}

The microtubule-associated protein tau modulates the extent and the rate of microtubule assembly (Weingarten et al., 1975). The underlying relationship between tau dysfunction and neurodegeneration was established when an inherited form of frontotemporal dementia with parkinsonism (FTDP-17) was linked to mutations in the MAPT gene located on chromosome 17, which codes for tau protein (Hutton et al., 1998; Poorkaj et al., 1998; Spillantini et al., 1998). Pathological tau protein modifications are critically involved in the pathogenesis of numerous nervous system disorders collectively known as tauopathies 
(Lee et al., 2001). Tauopathies, including Alzheimer's disease and FTDP-17, are characterized by tau hyperphosphorylation, detachment of tau from axonal microtubules and subsequent misfolding and aggregation. Pathological aggregates can be observed as neurofibrillary tangles (NFTs) in neuronal cell bodies and as neuropil threads within neuronal processes. The exact nature of neurotoxic tau protein species or species inducing neuronal malfunction is still not well understood (Ballatore et al., 2007). The classical view implies that NFTs are deleterious for neurons. This is corroborated by the numerous studies that showed that the number and extent of NFTs spread throughout the brain is positively correlated with the progression of neurodegeneration, neuronal death and cognitive decline in patients with Alzheimer's disease (Arriagada et al., 1992; Gómez-Isla et al., 1997; Nelson et al., 2012). On the other hand, studies in transgenic mouse models challenged this view. A seminal paper by SantaCruz and coauthors in conditional mouse model of tauopathy (rTg4510 mice) suggested that NFT formation can be dissociated from memory loss and neurodegeneration (SantaCruz et al., 2005).

Further, no link could be established between the presence of NFTs and structural and functional degeneration observed in frontal cortical pyramidal neurons (Rocher et al., 2010). The studies coming from the Hyman lab suggested that NFT-bearing neurons can survive for prolonged periods of time and they are functionally integrated in neuronal circuits even in the late stages of the disease (de Calignon et al., 2010; Kuchibhotla et al., 2014). Taken together, these studies point to the possible toxic role of tau species present before formation of mature NFTs: mainly still soluble, misfolded and hyperphosphorylated tau protein and probably to a lesser extent, early insoluble tau filaments (Berger et al., 2007).

Recent studies showed that injection of brain extracts containing tau fibrils or injection of synthetic tau fibrils into brains of tau transgenic mice induce NFT formation and spreading (Clavaguera et al., 2009; Iba et al., 2013). It was suggested that tau fibrils serve as seeds to induce templated tau aggregation. In addition, tau fibrils are most likely tau species that spread throughout the brain. These findings opened new avenues to study the effects of tau aggregates on neuronal structure and function. We wanted to take advantage of this new possibility and induce NFT formation in neurons and circuits that are normally not affected by this type of tau aggregates.

To investigate the effects of various pathological tau species on neuronal network functioning, we combined tau seeding with chronic in vivo two-photon calcium imaging in awake head-fixed mice expressing human tau with the P301S mutation (Allen et al., 2002; Andermann et al., 2010; Iba et al., 2013; Stancu et al., 2015). This approach enabled us to study the functional consequences of NFT formation in intact neuronal circuits and to directly compare the performance of NFT-bearing neurons with NFT- free neurons over the disease course. Our results suggest that soluble pathological tau species are responsible for the observed reduction in neuronal activity of cortical layer 2/3 neurons in P301S mice. NFT formation, induced by templated misfolding and aggregation of tau protein, did not induce additional deleterious effects in our assay. Observed reduction in neuronal activity of cortical layer 2/3 neurons in P301S mice did not show progression with disease course.

\section{Materials and methods}

\section{Animals}

We used homozygous transgenic male and female mice expressing human mutant P301S tau (383 aa four-repeat isoform of human tau) under the control of the Thy1.2 promoter (Allen et al., 2002) kindly provided to us by Dr Michel Goedert (Cambridge, UK). The P301S mice were backcrossed to $\mathrm{C} 57 \mathrm{BL} / 6 \mathrm{~J}$ line for at least nine generations to obtain animals on a pure C57BL/6J background. Transgene homozygosity was determined by real-time PCR. As control animals, we used mice of C57BL/6J strain (wild-type). The mice were housed under a 12-h light/dark cycle and were given food and water ad libitum. All procedures were performed in accordance with an animal protocol approved by the Government of Upper Bavaria (ref number GZ: 55.2-1-54-2532-163-13).

\section{Stereotaxic injections of tau preformed fibrils and virus}

Two-month-old wild-type or P301S mice were anaesthetized with an intraperitoneal injection of ketamine-xylazine (ketamine $140 \mathrm{mg} / \mathrm{kg}$, xylazine $10 \mathrm{mg} / \mathrm{kg}$ ). In addition, dexamethasone $(6 \mathrm{mg} / \mathrm{kg})$ was injected intraperitoneally to prevent the development of cerebral oedema. Mice were then placed on a heating blanket and stabilized in a stereotaxic frame. Tau preformed fibrils (tau-PFFs) were prepared as described previously (Stancu et al., 2015) and kindly provided to us by Dr Ilse Dewachter. Before injection, tau-PFFs were sonicated and mixed with AAV1.hSyn1.mRuby2.GSG.P2A. GCaMP6s.WPRE.SV4 virus (Rose et al., 2016; Cat. No. 50942-AAV1 Penn Vector Core). This AAV vector enables bicistronic stoichiometric expression of calcium indicator GCaMP6s and red fluorescent protein mRuby2 under a human synapsin 1 promoter.

Unilateral stereotaxic injection (right hemisphere) was performed in the frontal cortex $(\mathrm{A} / \mathrm{P},+2.0 \mathrm{~mm}$; $\mathrm{L},+1.4 \mathrm{~mm}$, $\mathrm{D} / \mathrm{V},-0.7 \mathrm{~mm}$ ). Total injected volume was $5 \mu \mathrm{l}$ (final tauPFFs concentration $333 \mu \mathrm{M}$, final virus titre $0.33 \times 10^{13}$ genome copies $(\mathrm{GC}) / \mathrm{ml})$. Vehicle without tau-PFFs $(5 \mu \mathrm{l})$ was injected in the control group. The mice were allocated to the experimental groups randomly and matching with littermates as controls was used.

\section{Implantation of cranial window}

Cranial window implantation was performed as described previously (Holtmaat et al., 2009). After stereotaxic tau-PFFs and 
virus injection a circular craniotomy was made using a dental drill (Schick-Technikmaster C1; Pluraden), and immediately covered with rounded coverslip ( $3 \mathrm{~mm}$ ).

The coverslip was sealed to the skull using dental acrylic (Cyano-Veneer fast; Schein). A custom made small metal bar was cemented next to the coverslip to allow head-fixation during training and imaging sessions. After surgery, mice received subcutaneous doses of the analgesic carprofen $(7.5 \mathrm{mg} /$ $\mathrm{kg})$ and the antibiotic cefotaxime $(5 \mathrm{mg} / \mathrm{kg})$. The mice with cranial window implanted were housed individually in cages.

\section{Imaging in awake, head-fixed mice}

Imaging was performed in awake, head-fixed mice as described previously (Andermann et al., 2010). Mice were trained for head-fixation for at least 7 days prior to imaging. On the first day of training, mice were handled to habituate to the researcher and to the set-up noise and they were allowed to explore and habituate to the holding tube. A separate holding tube was assigned to each mouse. On the next day, in addition to habituation, mice would be occasionally head-fixed after passing through the holding tube for a brief period of time $(10-30 \mathrm{~s})$. In the following days, periods of head-fixation were gradually increased up to $1 \mathrm{~h}$ at the end of training. Care was taken to make the mice feel comfortable in the holding tube and individual adjustments were made for each mouse if necessary. During head-fixation, mice typically showed long episodes of quiet wakefulness (quiet) interrupted by brief episodes of intensive whisking and movement (active). Therefore, we used whisking movement as an indicator of behavioural state (quiet versus active) (Crochet and Petersen, 2006).

Imaging started at least 20 days after surgery to allow mice to recover and cranial windows to become clear. Before each imaging session, mice were head-fixed and placed under the microscope for $5 \mathrm{~min}$ to habituate. Imaging was performed in the dark without any stimuli. In vivo time-lapse imaging series of spontaneous activity of layer $2 / 3$ frontal cortex neurons were acquired using a La Vision Trim Scope (La Vision BioTec) equipped with tunable Ti:sapphire two-photon lasers (Coherent Chameleon, Coherent and Mai Tai, Spectra Physics). The setup was controlled using La Vision Imspector software (La Vision BioTec). For recording calcium transients from layer $2 / 3$ neurons, the laser was tuned to $940 \mathrm{~nm}$, which enabled simultaneous excitation of mRuby 2 and GCaMP6s. To visualize NFTs in vivo we injected the fluorescent Congo red derivative FSB [(trans, trans)-1-fluoro-2,5-bis(3-hydroxycarbonyl-4-hydroxy)styrylbenzene] $24-48 \mathrm{~h}$ prior to imaging (Velasco et al., 2008; Schön et al., 2012). Mice were intraperitoneally injected with $300 \mu \mathrm{l}$ of $0.1 \%$ FSB $(2.4 \mathrm{mM}$, Cat. No. 344101-5MG, Merck) in PBS containing 2\% mouse albumin (Cat. No. 126674-25MG, Merck). When excited at 700$750 \mathrm{~nm}$ by two-photon laser, FSB provided robust and bright NFT labelling. To avoid photo-bleaching and photo-damage, laser power was kept under $80 \mathrm{~mW}$ at all times (measured at the back-focal plane of the objective). All time-lapse movies were taken at the frame rate of $>10 \mathrm{~Hz}$. For simultaneous imaging of NFTs and cortical layer $2 / 3$ neurons labelled with GCaMP6s/mRuby2, Mai Tai tuned to $700-750 \mathrm{~nm}$ and Chameleon tuned to $940 \mathrm{~nm}$ were used in alternate laser scan protocol. Emitted fluorescence was split at $495 \mathrm{~nm}$ and $560 \mathrm{~nm}$ and detected by photomultiplier tubes. Figures $1 \mathrm{E}$ and $2 \mathrm{~A}$ represent $>50$ time-series images acquired at $>10 \mathrm{~Hz}$ and averaged. For representation purposes in Figs 1E and 2A, images were combined using 'screen' function in Photoshop software (Adobe) and gamma was adjusted non-linearly. For recording mouse behaviour, a web camera was installed and controlled by La Vison Imspector software, which allowed synchronous recordings with the same frame rate for mouse behaviour and calcium transients.

Mice were imaged in repeated sessions up to $7(\sim 50$ days $)$ weeks after seeding. Only datasets from mice with more than three imaging sessions in total were analysed further. A single imaging session would typically last around $45 \mathrm{~min}$. During this time period, images from three to four areas of interest were acquired ( $\sim 10 \mathrm{~min}$ per area, 50-150 neurons per area).

\section{Immunohistochemistry, confocal microscopy and image processing}

Mice were perfused transcardially with PBS followed by $4 \%$ paraformaldehyde (PFA) in PBS. Brains were removed and fixed overnight in $4 \%$ PFA in PBS at $4^{\circ} \mathrm{C}$. Fifty micrometrethick free-floating brain sagittal section were cut using a microtome. The sections were incubated in $10 \%$ normal goat serum (Sigma-Aldrich), $1 \%$ bovine serum albumin, and $0.1 \%$ Triton $^{\mathrm{TM}}$ X-100 in PBS and stained with AT8 antibody recognizing human and murine tau phosphorylated at S202 and T205 (Thermo Fisher Scientific Cat. No. MN1020). Images were acquired with a LSM 780 confocal microscope (Zeiss) equipped with a $40 \times / 1.4$ oil immersion objective. For representation in Fig. 1A-D, spectral channels of confocal maximum intensity projections were combined in pseudocolour by using the 'screen' function in Photoshop (Adobe) software. Gamma was adjusted non-linearly to show low-intensity objects.

\section{Data processing and image analysis}

Collected images were processed and analysed using custom written codes in MATLAB and ImageJ software. A typical recording consisted of two image series simultaneously recorded using two-photon microscopy. One series contained cell bodies labelled with mRuby2 (calcium insensitive, red channel, $>560 \mathrm{~nm}$ ). The other series contained fluorescence of the cytoplasmic calcium indicator GCaMP6s (green channel, 495-560 nm). Behavioural state was recorded by a camera focused at the whiskers and classified as active or quiescent (quiet) using custom written MATLAB code. A behaviour would be classified as quiet or active if it persisted for $\geqslant 7$ frames $(0.7 \mathrm{~s})$.

Two-photon calcium imaging data were analysed using custom written MATLAB code. In brief, individual images from a single time series were aligned to each other in the $x-y$ plane. Image registration parameters ( $x / y$ shift) were estimated using a Fourier transform based approach (Guizar-Sicairos et al., 2008) on image data from the mRuby2 (structural) channel (Rose et al., 2016). Regions of interest (regions of interest) were manually identified using custom written software in MATLAB. For each region of interest, a raw GCaMP6s fluorescence time series was constructed by averaging the pixel values within the region of interest for each individual imaging frame.

Time series were corrected for contamination by local neuropil fluorescence and for changes in fluorescence caused 

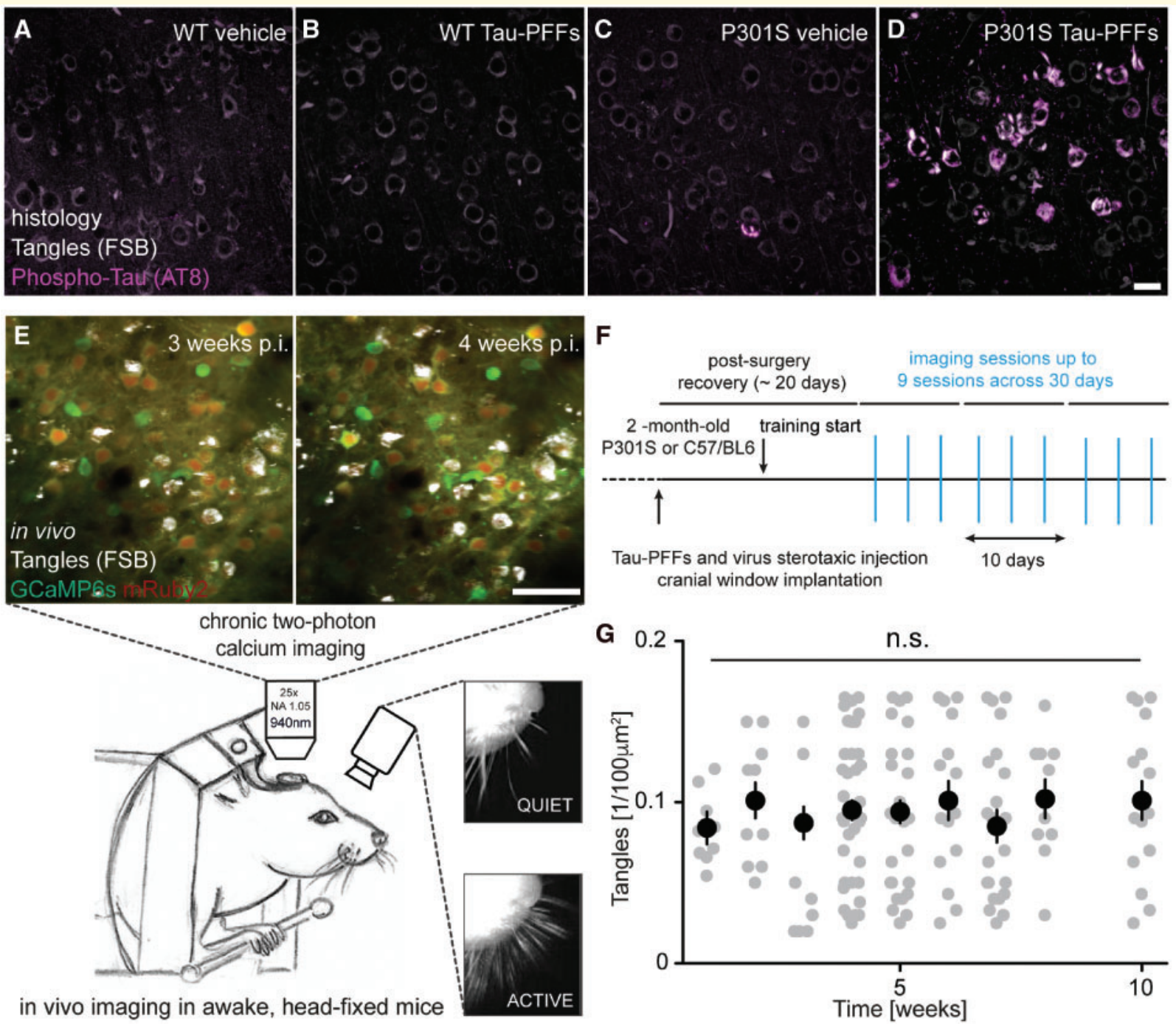

Figure I Injection of tau-PFFs induces rapid formation of NFTs in P30 IS mice. (A-D) Frontal cortex of a wild-type mouse 3 weeks after injection with vehicle (A), with tau-PFFs (B) and a P30IS homozygous mouse 3 weeks after injection with vehicle (C), with tau-PFFs (D) stained with FSB (white) and AT8 antibody (magenta). All mice were injected at 2 months of age. Scale bar $=20 \mu \mathrm{m}$. (E) Top: Principle of chronic two-photon calcium imaging in tauopathy model. AAVI transduced neurons are labelled with mRuby2 (red) and GCaMP6s (green). NFTs are labelled with FSB (white). The same neuronal population can be found over time [3 weeks post-injection (p.i.) left versus 4 weeks postinjection right]. Images are made by averaging $>50$ time-series frames acquired in vivo at $>10 \mathrm{~Hz}$ with two-photon lasers tuned to $940 \mathrm{~nm}$ for GCaMP6s/mRuby2 and to $750 \mathrm{~nm}$ for FSB. Bottom: Schematic of a head-fixed mouse in the experimental setup with the camera for recording of behaviour. Scale bar $=50 \mu \mathrm{m}$. (F) Schematic of the experimental protocol. (G) Density of NFTs over time. Black circles represent mean values \pm SEM. Grey values represent individual fields of view ( $n=9$ mice, 53 areas imaged, not significant, one-way ANOVA).

by movement along the $z$-axis (in/out of plane movement) using the following method. First, local neuropil fluorescence was calculated by averaging all pixel values in a $\sim 30-\mu \mathrm{m}$ ring around the region of interest, excluding other regions of interest. Next, the effect of $z$-movement on the fluorescence intensity of a region of interest was determined from the mRuby2 fluorescence within the region of interest. Using linear regression (Equation 1) we could now infer the contribution of the local neuropil signal $\left(F_{\text {neuropil }}\right)$ and z-motion $\left(F_{\text {motion }}\right)$ to the fluorescence within the region of interest $\left(F_{R O I_{\text {raw }}}\right)$. Finally, the resulting corrected time series
$\left(F_{R O I_{\text {corrected }}}\right)$ was readjusted to the median of the original time series $\left(F_{R O I_{\text {raw }}}\right)$.

$$
F_{R O I_{\text {raw }}}=F_{R O I_{\text {corrected }}}+a \cdot F_{\text {neuropil }}+b \cdot F_{\text {motion }}
$$

The relative fluorescence change over baseline, $\Delta \mathrm{F} / \mathrm{F}$, was calculated according to Equation 2, where $F_{t}$ is the absolute fluorescence at imaging frame $t$. Baseline fluorescence $F_{0_{t}}$ was calculated using a sliding window approach by, for each frame, averaging the lowest $50 \%$ fluorescence values in a 30 -s sliding window before imaging frame $t$ (Kerr and Denk, 2008). 


$$
\Delta F_{t}=\frac{F_{t}-F_{0 t}}{F_{0 t}}
$$

Transients were detected as peaks in the fluorescent time series that exceeded $>3.5$ standard deviations $(\mathrm{SD})$ of the overall time series, had a rising time of $0.2 \mathrm{~s}$, decay time of $0.5 \mathrm{~s}$ and interpeak interval $>0.7 \mathrm{~s}$.

Correlations between time series of $\Delta \mathrm{F} / \mathrm{F}$ calcium activity were calculated using the Pearson correlation coefficient on $\mathrm{z}$-scored segments and then averaged.

\section{Statistics}

Statistical analysis was performed using one-way ANOVA, two-way ANOVA, multivariate analysis of variance (MANOVA) and Student's $t$-tests with GraphPad Prism and STATISTICA software. $P$-values are reported and outlined as follows: $\quad * P<0.05, \quad * * P<0.01, \quad * * P<0.001 \quad$ and $* * * P<0.0001$. The mean and standard error of the mean (SEM) are indicated.

\section{Data availability}

Raw data were generated at Center for Neuropathology and Prion Research, Ludwig-Maximilians-University Munich, Munich, Germany. Derived data supporting the findings of this study are available from the corresponding author on request.

\section{Results}

\section{Injection of tau preformed fibrilss induces rapid formation of NFTs in P30IS mice}

Using histological analysis, we did not observe formation of NFTs in the frontal cortex of wild-type mice stereotactically injected with vehicle or tau-PFFs, (Fig. $1 \mathrm{~A}$ and B). Similarly, histological analysis revealed no formation of NFTs in P301S mice injected stereotactically with vehicle (Fig. 1C). However, injection of tau-PFFs in P301S mice caused rapid and robust formation of tau aggregates in the frontal cortex. These aggregates were consisting of hyperphosphorylated (AT8-positive) and fibrillar tau (FSBpositive) (Fig. 1D). Additional Bielschowsky staining confirmed that these structures are silver positive and therefore can be considered as NFTs (data not shown).

Next, we aimed to detect NFTs in vivo using cranial window preparation. Typically, in our experiments, it takes $\sim 3$ weeks after surgery for the cranial window to become clear for in vivo imaging. At this time point, using intraperitoneal injections of FSB dye, NFTs were easily detectible in vivo in the frontal cortex of P301S mice injected with tau-PFFs (Fig. 1E). This gave us an excellent opportunity to investigate the functional consequences of NFT formation at the single neuron level using two-photon in vivo calcium imaging (Fig. 1E). We were able to repetitively record over time from the same neuronal populations and follow individual neurons (Fig. 1E). Furthermore, using FSB staining we could easily differentiate between the tangle-bearing neurons (NFTbearing) and their tangle-free neighbours (NFT- free). Our experimental design (Fig. 1F) allowed us to record from 4580 neurons over three or more time points in a total of 23 mice. Of tracked neurons, 410 of the 4580 were NFT-bearing.

To investigate how fast the NFTs formed in our model and to probe whether novel NFTs could form over time, we used intraperitoneal injections of FSB to label all the NFTs formed at a given time point. We performed this experiment in a separate cohort of mice that we followed up to 10 weeks after tau-PFF injection (Fig. 1G). In this way, we were able to compare the number and presence of NFTs in the same area (field of view) over time. Surprisingly, in vivo two-photon imaging revealed that NFTs had already formed at 1 week after injection. However, the number of NFTs did not change significantly over time (Fig. 1G). In rare cases, we could observe the formation of novel NFTs [in 7 of 53 areas $(13.2 \%)$ imaged in nine mice, totalling eight new NFTs]. All of these NFT formation events occurred within the first 3 weeks after injection, i.e. when functional imaging is still not possible because of limited clarity of the cranial window. No NFTs disappeared during imaging period.

\section{Neuronal activity is reduced in P30IS mice independently of presence of NFTs}

To investigate functional consequences of NFT formation we used two-photon calcium imaging in awake head-fixed mice. We were able to simultaneously record calcium transients from 50-150 neurons in one field of view (Fig. 2A). During both quiet and active behavioural states, neurons in P301S mice showed a clear reduction of activity measured by the number of calcium transients per minute (Fig. 2B). Tested by two-way ANOVA, we found a significant effect of the main factor genotype [wild-type versus P301S: $F(1,19)=15.61, P<0.001]$. Effects of injection and interaction were not significant: (vehicle versus tau-PFFs) $F(1,19)=0,05, P=0.83$ and $F(1,19)=0.16, P=0.69$, respectively. Closer analysis revealed no difference between NFT-bearing neurons and their NFT-free neighbours (Fig. 2B, red triangles versus red squares). Similar results were obtained when we analysed activity during active phases. Again, only effect of genotype was significant: $F(1,19)=27.79, P<0.0001$. Effects of injection (vehicle versus tau-PFFs) and interaction were not significant: $F(1,19)=0.6454, \quad P=0.4317$ and $F(1,19)=0.01604$, $P=0.9006$, respectively. Similar to the quiet state, no difference in transient frequency between NFT-bearing neurons and their NFT-free neighbours was observed during the active state (Fig. 2B). To present the distribution of 
A
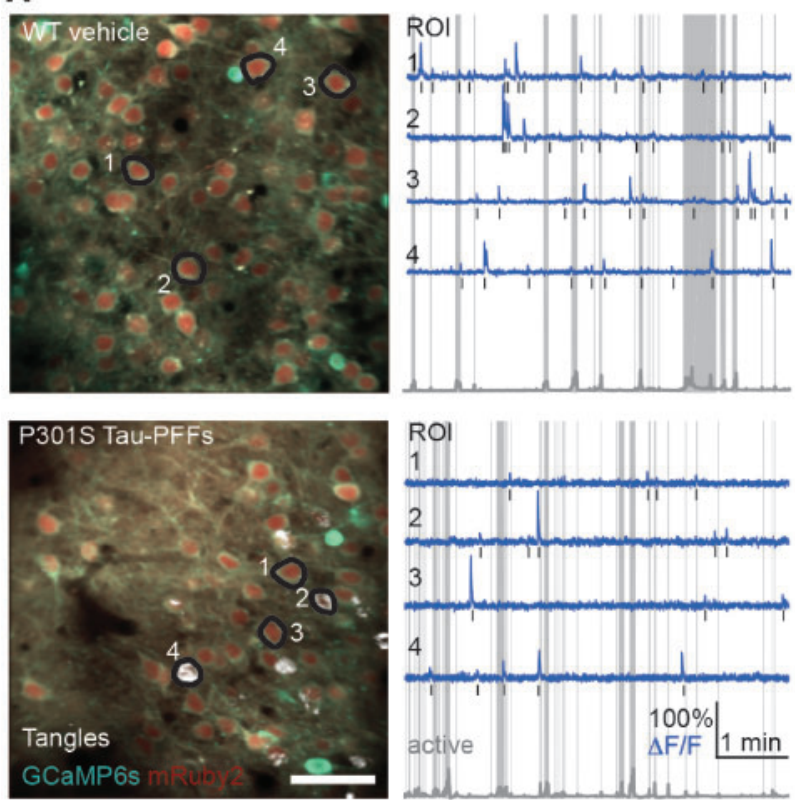

\section{B o WT vehicle oP301S vehicle $\quad$ NFT-free O WT Tau-PFFs P P301S Tau-PFFs $\Delta$ NFT-bearing}
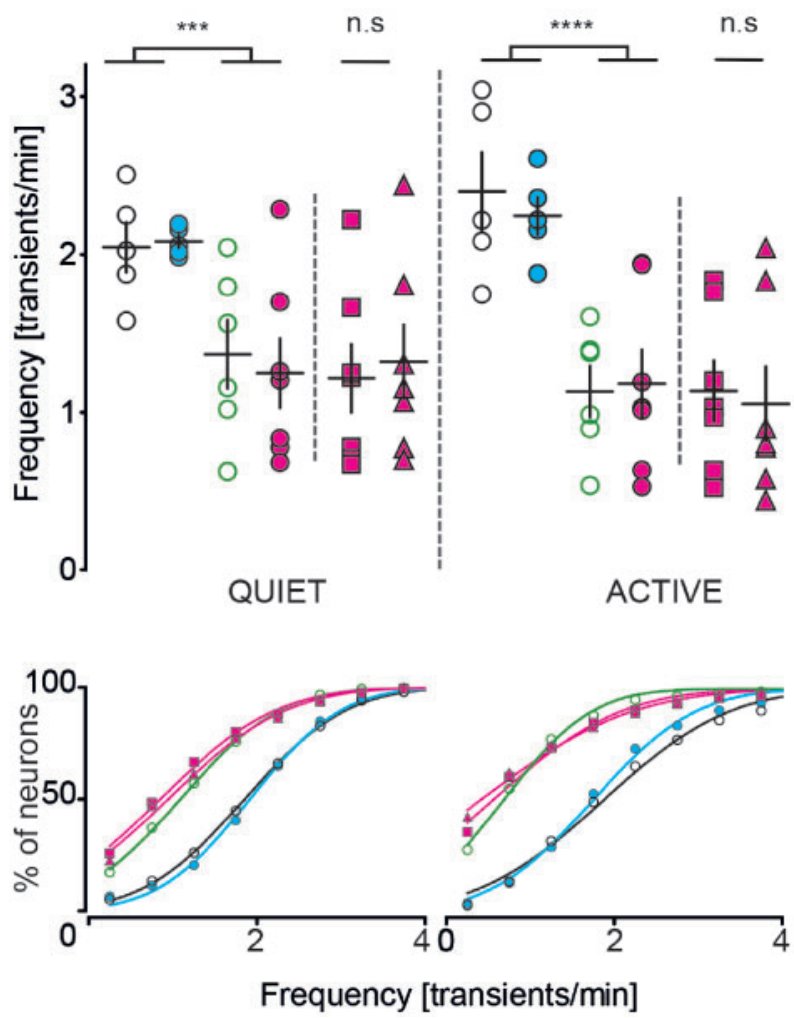

Figure 2 Neuronal activity is reduced in P30IS mice independently of presence of NFTs. (A) Left: Representative in vivo recordings from wild-type vehicle and P30IS tau-PFFs mice. AAVI transduced neurons are labelled with mRuby2 (red) and GCaMP6s (green). NFTs are labelled with FSB (white). Images are made by averaging $>50$ time-series frames acquired in vivo at $>10 \mathrm{~Hz}$ with two-photon lasers tuned to $940 \mathrm{~nm}$ for CGaMP6s/mRuby2 and to $750 \mathrm{~nm}$ for FSB. Scale bar $=50 \mu \mathrm{m}$. Right: Traces (blue) extracted from annotated regions of interest (black) during quiet and active (grey shade) behavioural states classified based on changes in whisking movement (grey trace in the bottom). Note that traces 2 and 4 in P30IS tau-PFFs group are from NFT-bearing neurons. Black bars mark detected calcium transients. (B) Top: Mean frequency of calcium transients during quiet state of all neurons detectable in three or more time points. Wild-type vehicle (WT) (black), wild-type tau-PFFs (cyan) P30IS vehicle (green), P30IS tau-PFFs: all neurons are denoted as magenta circles, with NFT-free as magenta squares and NFT-bearing as magenta triangles. Data points represent individual mice, $n=5-7$ mice per group; black lines represent mean value $\pm S E M$. ***P $<0.00$ I, $* * * * P<0.000$ I, wild-type versus P30 IS [two-way ANOVA, genotype factor, not significant (Student's $t$-test)]. Bottom: Cumulative frequency plots of the data shown in the top panel. 
frequencies of individual neurons we plotted the results as cumulative frequency graphs. In both quiet and active states a clear shift to the left of the P301S curves is evident, indicating lower activity (Fig. 2). No difference was observed between NFT-bearing and NFT-free neurons. Next, we investigated if the duration and amplitude of the transients is different between the groups. To analyse this, we calculated the area under the curve (AUC) normalized to the duration of transients (i.e. AUC/s, Supplementary Fig. 1). The results of AUC analysis were similar to the results of frequency analysis. Tested by twoway ANOVA we found significant main factor of genotype [wild-type versus P301S: $F(1,19)=12,79, P<0.01$ ]. Effects of injection and interaction were not significant: (vehicle versus tau-PFFs), $\quad F(1,19)=0.16, \quad P=0.86 \quad$ and $F(1,19)=0.04, P=0.43$, respectively. Closer analysis revealed no difference between NFT-bearing neurons and their NFT-free neighbours (Supplementary Fig. 1).

\section{Reduced neuronal activity in P30IS mice does not change with time and pathology progression}

To investigate how activity of the neuronal ensembles changes over time we calculated the percentage of neurons with low activity $(<1$ transient/min) and high activity $(>3$ transient/min) averaged in 10-day bins over the whole imaging period (up to 50 days after seeding). Interestingly, in all groups the distribution of neurons according to their

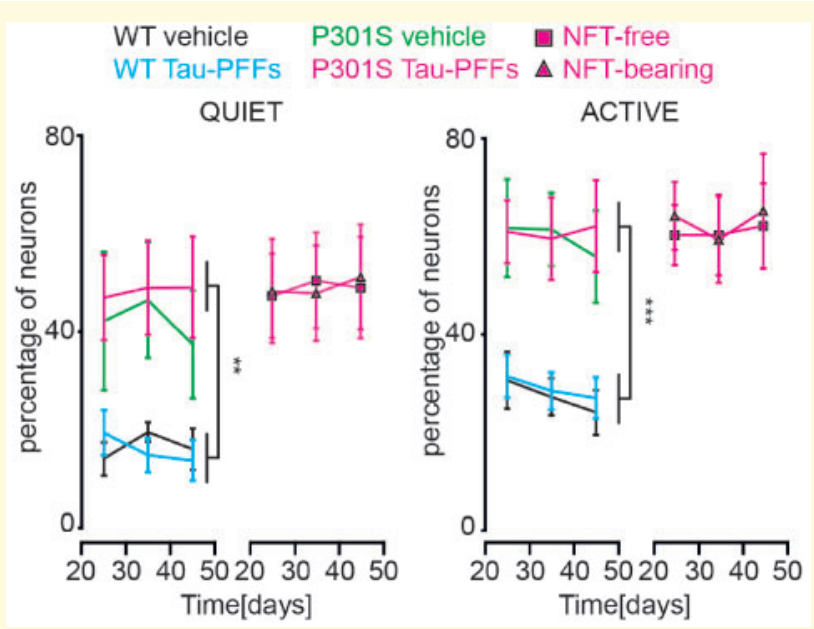

Figure 3 Reduced neuronal activity in P30 IS mice does not change with time and pathology progression. Average fraction (\% mean \pm SEM) of neurons with low activity $(<I$ transients/min) during quiet state (left graph) and active state (right graph) averaged within 10-day time bins over whole experimental period. **P $<0.0$ I wild-type versus P30IS (MANOVA, genotype factor) $* * * P<0.001$ wild-type versus P30IS (MANOVA, genotype factor). Wild-type vehicle (black), wild-type tau-PFFs (cyan) P30IS vehicle (green), P30IS tau-PFFs: all neurons in magenta, with NFT-free neurons as magenta squares and NFT-bearing neurons as magenta triangles. activity in both quiet and active phases was similar over time. (Fig. 3 and Supplementary Fig. 2). Both wild-type groups (wild-type vehicle and wild-type tau-PFFs) had a relatively small fraction of neurons with low activity and much larger fraction of neurons with high activity, both during quiet and active phases (Fig. 3 and Supplementary Fig. 2). In contrast, P301S mice, both injected with vehicle and tau-PFFs had a large fraction of neurons with low activity and a small fraction of neurons with high activity (Fig. 3 and Supplementary Fig. 2). When we only considered NFT-bearing neurons we did not detect significant changes in the distribution of neurons according to their activity during quiet and active phases. This group of NFTbearing neurons, similar to NFT-free neurons, had no significant change in fractions of neurons with low and high activity over time (Fig. 3 and Supplementary Fig. 2).

Although the distribution of neurons according to their activity seems to be constant over time, it is still possible that individual neurons significantly change their activity frequency from one time-point to another. For example, a neuron could become highly active and, in the same time, another neuron could become hypoactive without affecting the overall population distribution. To investigate this in more detail, we calculated the average absolute change in activity between time points for each individual neuron in the early phase after injection ( $<35$ days post-injection) and in the late phase ( $>35$ days post-injection). Our results show that individual neurons undergo comparable absolute activity changes from one imaging point to the other in early and in late phase after seeding (Supplementary Fig. 3).

\section{Correlation coefficients in imaged neuronal populations remain stable over time}

Finally, we assessed how strong the interactions in imaged local neuronal populations were. For each neuronal population (i.e. each field of view) we calculated the average correlation coefficient of all pairs of imaged neurons. This analysis revealed that, in contrast to the frequency of spontaneous activity, the average correlation coefficient was not different among the groups (Fig. 4A). The correlation coefficient values were comparable among the groups when calculated for entire recordings (quiet and active together), as well as when calculated separately using segments for only quiet or only active phases. In mice injected with tau-PFFs, we calculated the average correlation coefficient between only NFT-free, only NFT-bearing or pairing NFTfree with NFT-bearing neurons. Interestingly, no difference was observed among these groups as well (Fig. 4B).

The underlying connectivity within a given neuronal population can be indirectly inferred from the pattern of correlations in recorded calcium dynamics (Ko et al., 2011). If the present pathology induced progressive changes of such connectivity, this would be reflected in alterations of correlation patterns over time. 

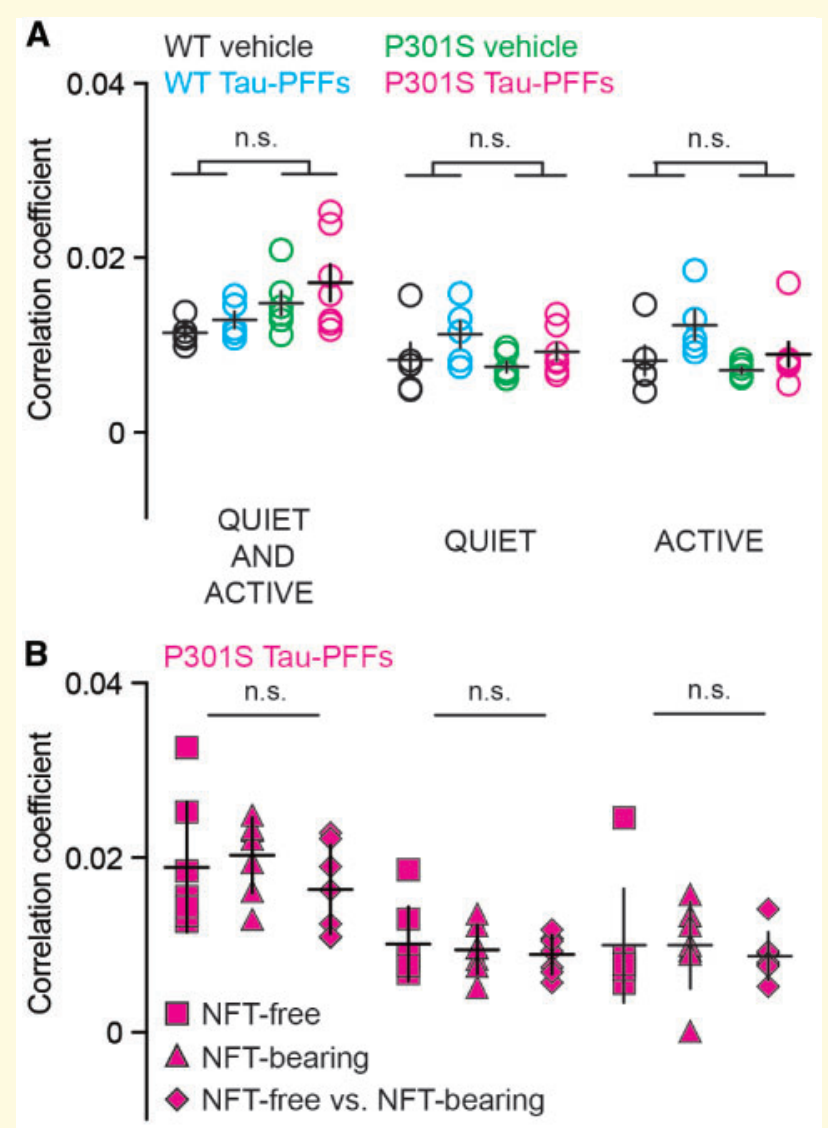

Figure 4 Correlation coefficients in imaged neuronal populations are comparable among the groups. (A) Pearson correlation coefficient (mean \pm SEM) calculated for each imaged field of view for entire recording (quiet and active), and separately for quiet and active states. Wild-type vehicle (black), wild-type tauPFFs (cyan) P30IS vehicle (green), P30IS tau-PFFs (magenta). Data points represent individual mice, $n=5-7$ mice per group; black lines represent mean value \pm SEM (not significant, two-way ANOVA). (B) Pearson correlation coefficient (mean \pm SEM) calculated for each imaged field of view for entire recording (quiet and active), and separately for quiet and active states for P30IS tau-PFFs group. Correlations were calculated including only NFT-free neurons (magenta squares), only P30IS tau-PFFs NFT-bearing neurons (magenta triangles) or only between NFT-free neurons and NFTbearing neurons (magenta diamonds). Data points represent individual mice; $n=5-7$ mice per group; black lines represent mean value $\pm \operatorname{SEM}$ (n.s. one-way ANOVA).

To assess this, the pattern of an individual time point was defined as all pairwise correlations from an imaged neuronal population. Next, to evaluate the pattern change between a given two time points we calculated how strongly the pattern of correlations persisted (expressed as correlation of the pattern across time points) and averaged within 10-day time bins (Fig. 5A). The analysis showed that despite existing pathology, the pattern of neuronal pairwise correlations remained stable over time in all groups and for the different behaviours (i.e. quite and active).
To illustrate and visualize the pattern in the correlation coefficient matrix for individual neuronal populations, we sorted the neurons into four clusters exhibiting similar calcium activity patterns (using k-means clustering) in all experimental groups (Fig. 5B). Examples of correlation matrices for four time points are shown, highlighting that some pairwise correlations are persistent while others are lost over time.

More detailed analysis in tau-PFFs group showed that when analysed separately for NFT-free, NFT-bearing and NFT-free versus NFT-bearing neurons, correlation coefficient values were comparable among all groups and showed no changes over time (Fig. 5C and D). Taken together, the analysis of correlation patterns over time suggests that presence of pathological tau does not induce progressive changes in connectivity of investigated neuronal circuits.

\section{Discussion}

To assess the impact of pathological tau species on neuronal functioning, we combined injections of tau-PFFs with chronic in vivo two-photon calcium microscopy in a tauoverexpressing mouse model. This approach enabled us to directly compare the effects of soluble pathological tau with the effects of NFTs formation on the activity of individual neurons and neuronal circuits. In addition, by recording ongoing cortical activity in the same neuronal population repetitively over a prolonged period of time, we were able to mimic, to some extent, disease conditions in which pathological changes take place gradually over the disease course.

This study was conducted in P301S mice, which is a wellcharacterized mouse model that exhibits the essential features of a human tauopathy (Allen et al., 2002). In the neurons of these mice, human tau protein and undergoes well-defined time course of phosphorylation, misfolding, oligomerization and building of filamentous aggregates (Delobel et al., 2008). We deliberately chose to use P301S transgenic mice, since only a small subset of frontal cortex neurons contains filamentous tau (Fig. 1C and Hoffmann et al., 2013). However, upon injection of tauPFFs, these mice show rapid formation of NFTs that could be readily detected in vivo using FSB fluorescent dye (Velasco et al., 2008; Schön et al., 2012). In this study, we used synthetic pre-aggregated tau fragments, containing the four-repeat domain of TauP301L-tau-PFFs (Stancu et al., 2015). Therefore, there is the possibility that the observed tau aggregates might simply represent passive uptake of injected fibrils and not result from templated misfolding and aggregation of cell-endogenous tau. Two lines of evidence argue against such a possibility. The aggregates are strongly stained with anti-tau P-S202/T205 antibody (AT8). The AT8 epitope is not present on tauPFFs used for injection and therefore the aggregates that we observed derive, at least partially, from full-length 
A QUIET AND ACTIVE

QUIET

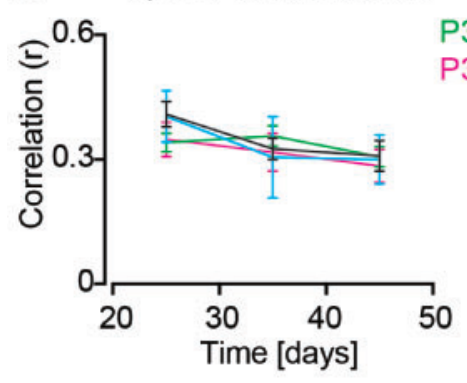

P301S vehicle
P301S Tau-PFFs

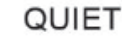

B

24 days p.i.

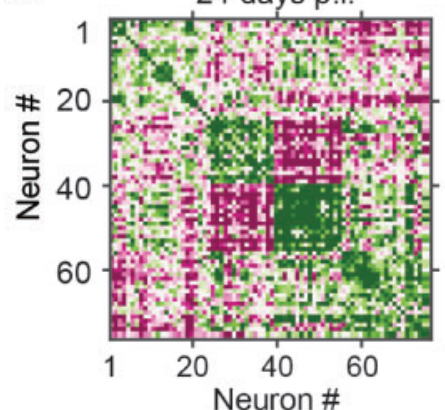

WT vehicle

\section{ACTIVE}

WT Tau-PFFs
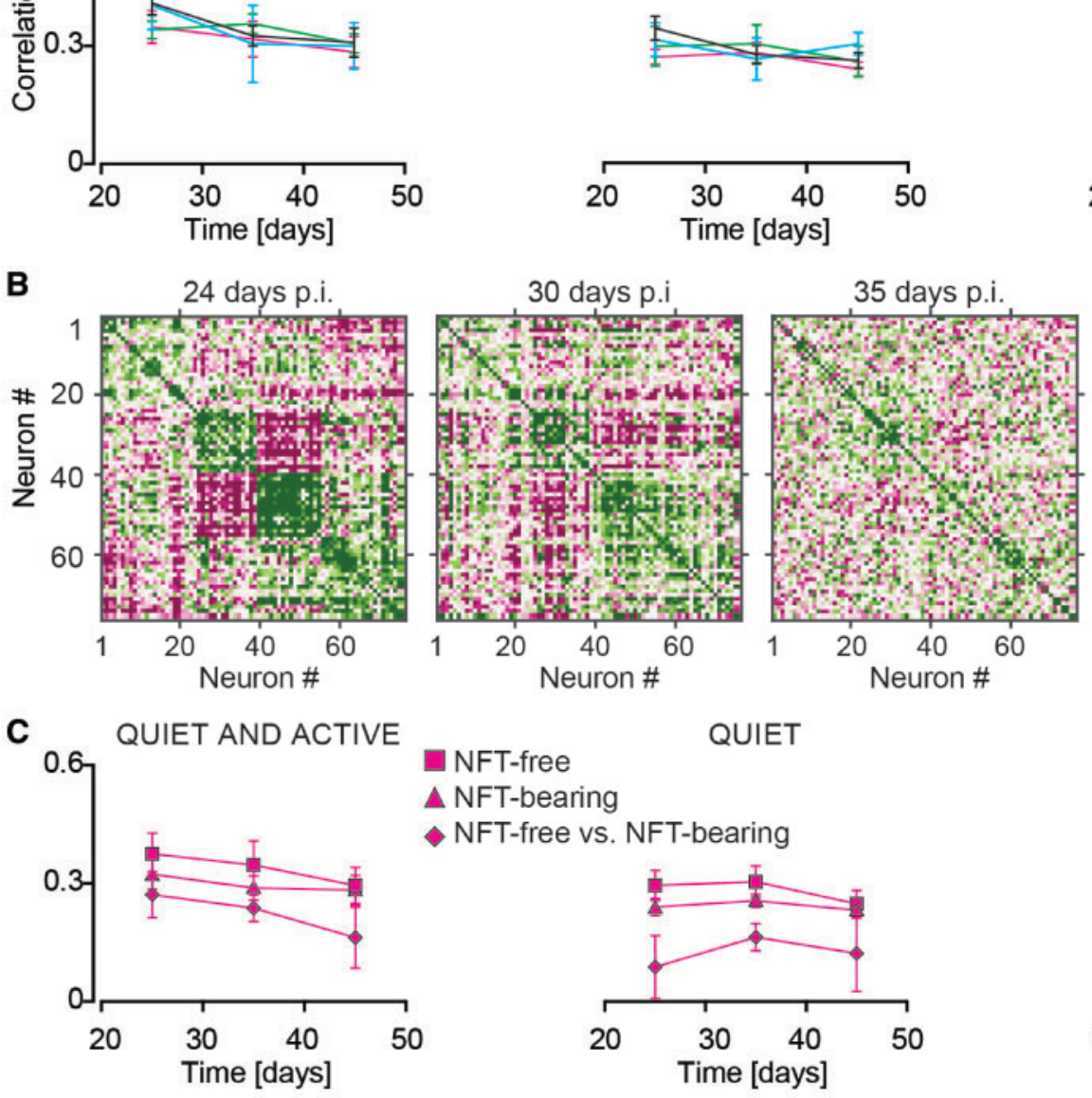

NFT-free

QUIET

$\Delta$ NFT-bearing

- NFT-free vs. NFT-bearing

D

21 days p.i.

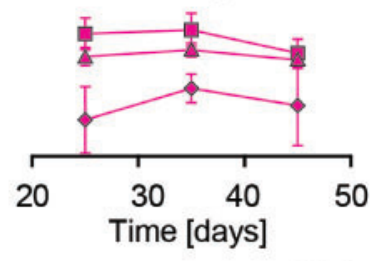

25 days p.i.
29 days p.i.
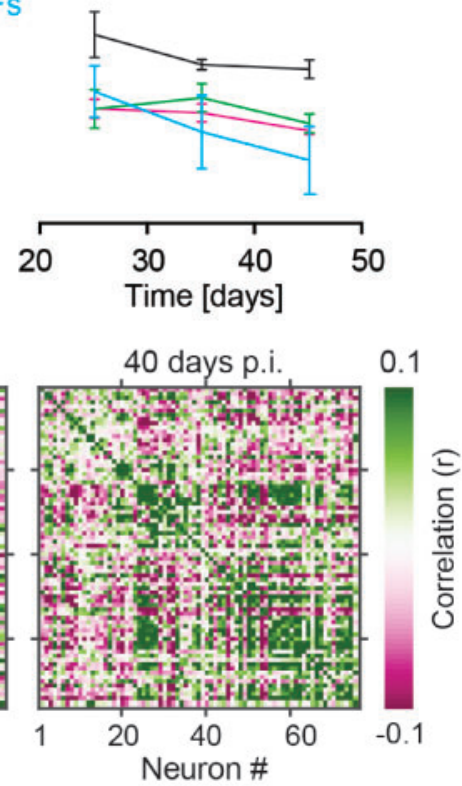

ACTIVE

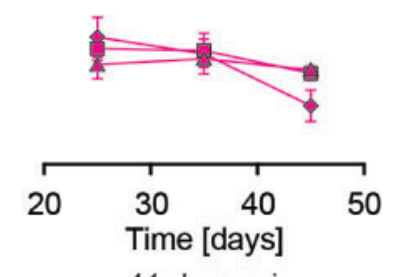

41 days p.i.

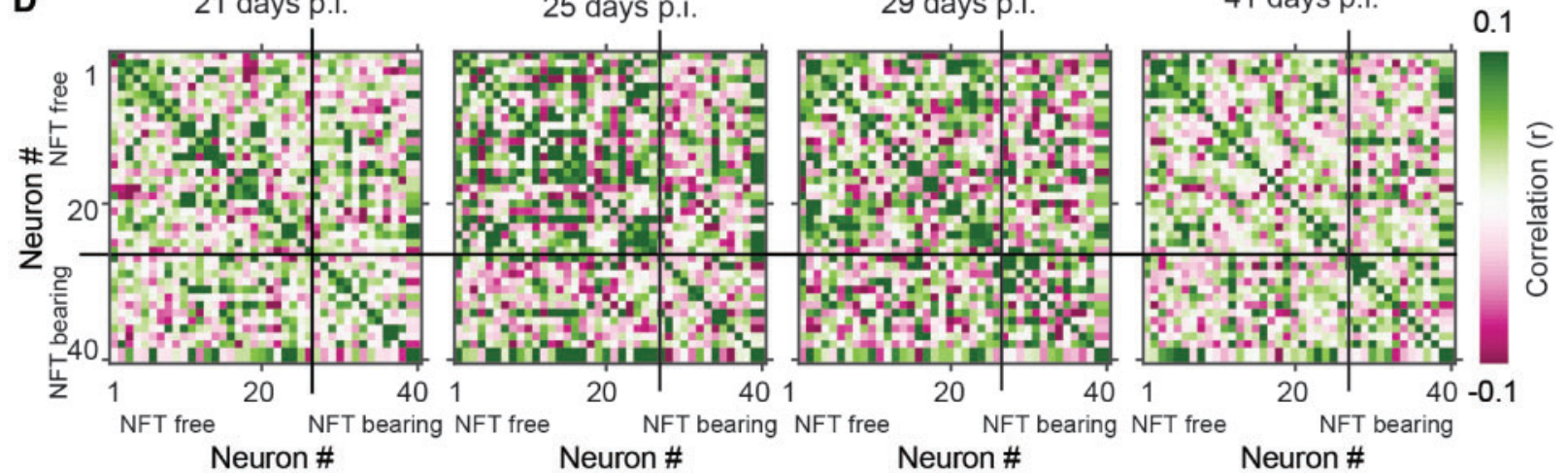

Figure 5 Correlation coefficients in imaged neuronal populations remain stable over time. (A) The change of the pairwise correlation pattern from an imaged neuronal population over whole experimental period. Correlation change for a given neuronal population was expressed as correlation patterns of different time points and averaged within 10-day time bins (mean \pm SEM). Correlation change was calculated using recordings from active and quiet phases together (left), and separately for quiet (middle) and active phase (right). Wild-type vehicle (black), wild-type tau-PFFs (cyan) P30IS vehicle (green), P30IS tau-PFFs (magenta). (B) The pattern in the correlation coefficient matrix for individual neuronal populations in wild-type experimental group. Neurons were sorted into four clusters exhibiting similar calcium activity patterns using kmeans clustering. Examples of correlation matrices for four time-points are shown (24, 30, 35 and 40 days post-injection). (C) The change of the pairwise correlation pattern from an imaged neuronal population over the whole experimental period in the P30IS tau-PFF experimental group. Correlation changes for NFT-free neurons (magenta squares), NFT-bearing (magenta triangles) and NFT-free versus NFT-bearing neurons were calculated separately (magenta diamonds) and expressed as correlation across time points and averaged within I0-day time bins (mean \pm SEM). Correlation change was calculated taking recordings from active and quiet phases together (left), and separately for quiet (middle) and active phase (right). (D) As in B, but using data of an example P30IS mouse. Black line distinguishes the subpopulation of NTF-bearing from NFT-free neurons. Examples of correlation matrices for four time points are shown (2I, 25, 29 and $4 \mathrm{I}$ days post-injection). p.i. = post-injection; WT = wild-type. 
endogenously expressed human tau. In addition, neurons in wild-type mice do not accumulate tau upon injection of tau-PFFs further opposing the passive uptake possibility.

To investigate the formation of novel NFTs in the frontal cortex over time, we imaged the mice in vivo up to 10 weeks after tau-PFF injection. Interestingly, we did not observe significant formation of de novo NFTs during this period, suggesting that mechanisms of local spreading are not present in our model. Indeed, at the injection site, all the NFTs formed within the first week after tau-PFF injection. We believe that rare cases in which we observed appearance of NFTs in our images are rather due to FSB accumulation with repeated injections and/or improved clarity of cranial window. Previous studies mainly used histological analysis to assess the level of NFTs spreading upon injection of seeding material (tau-PFFs or brain homogenates containing the tau fibrils). In all these studies a relatively slow progression ( $>6$ months) of NFTs was reported and predominately in anatomically connected brain regions (Clavaguera et al., 2009; Iba et al., 2013; Peeraer et al., 2015). Therefore, it is still possible that in our mice too, novel NFTs would appear after prolonged periods of time. Unfortunately, this is not possible to study in homozygote P301S mice because this line develops severe motor deficits with 5-6 months of age due to strong expression of the transgene in the brainstem (Allen et al., 2002). These results also raise an important question of careful titration of injected tau-PFF dose and appropriate choice of recipient mouse line to optimally mimic the templated misfolding and spreading observed in human patients.

Our in vivo recordings indicate clear overall reduction in spontaneous activity of layer $2 / 3$ neurons in P301S mice compared with wild-type mice. The P301S mouse model provides a unique opportunity to study three neuronal populations at the same time. The majority of cortical neurons overexpress human mutated tau protein, which is mislocalized in their somata (recognized by phophorylation independent HT7 antibody, Hoffmann et al., 2013). Few neurons in the cortex have pathologically phosphorylated tau and filamentous tau aggregates (AT8- and FSB-positive, respectively; Fig. 1C and Hoffmann et al., 2013). Finally, due to stochastic expression driven by the murine Thy 1.2 promoter, there is also a small subset of neurons that do not express the transgene $(<20 \%$, data not shown; Marinković et al., 2015). Our results clearly show that the presence of pathological mutated tau in the majority of neurons is sufficient to reduce the activity of the entire imaged neuronal population. A central goal of this study was to address whether introduction of additional and possibly also toxic tau species in the form of NFT had a specific impact on neuronal circuits. Interestingly, after injection of tau-PFFs in P301S mice and subsequent formation of NFTs in a subset of neurons, neuronal circuits showed spontaneous activity rates comparable to the circuits in P301S mice injected with the buffer. Specifically, when analysed at the single neuron level, the NFT-bearing neurons had similar calcium transient frequencies during both quiet and active phases as compared to their NFTfree neighbouring neurons. This indicates that formation of NFTs in neurons do not induce additional effects in neuronal functioning in our model. Using our chronic in vivo imaging approach, we were able for the first time to follow the effects investigate impact of soluble versus fibrillar tau in same individual neurons over prolonged periods of time. Interestingly, no significant changes in distribution of neurons according to their activity or correlation patterns could be detected in imaged circuits over the whole time course of our experiment ( $\sim 50$ days after seeding). This was surprising as we expected an emergence of progressive alterations due to the strong presence of pathological tau and ongoing neurodegeneration in CNS. This finding might suggest that there is remaining intrinsic capacity of circuits to compensate and maintain their function. However, we cannot exclude that subtle structural and functional changes take place which cannot be detected in our assay. It is also important to keep in mind that calcium transients do not fully represent neuronal functioning and that possible functional changes could have been detected by more suitable techniques like patch-clamp recordings. Alternatively, or additionally, it is possible that significant changes in neuronal activity develop after longer periods of time than in our experiment.

Numerous studies tried to elucidate the effects of NFTs formation on neuronal activity. Of interest for our work are those studies that investigated this matter at the single neuron level and which differentiated between the NFTbearing and NFT-free neurons. Elegant studies coming from the Lübke lab investigated this in great detail in cortical neurons using acute slice preparation (Rocher et al., 2010). Their results indicated that structural and functional changes in tau mutant mice are not linked to the presence of NFTs. They reported increased activity of neurons in transgenic mice in response to stimulation. An in vivo study from the Hyman lab showed that NFT-bearing neurons are functionally intact and do not impair local circuits (Kuchibhotla et al., 2014). Kuchibhotla and co-authors show that NFT-bearing and NFT-free neurons preserve their resting calcium levels and neuronal tuning. They also report that NFT-bearing and NFT-free neurons have comparable characteristics to the neurons in wild-type control group, which is different from our findings. Finally, relevant to the work here is a recent in vivo study that investigated ongoing activity in cortical neurons in intact circuits with significant NFT load (Menkes-Caspi et al., 2015). Similar to our results, this study revealed an overall reduction in the neuronal spontaneous activity confirming the disrupting role of the pathological tau protein. Since the authors did not differentiate between cortical neurons based on NFT presence it cannot be concluded which tau species is responsible for the observed effect.

All these studies were conducted in $\mathrm{rTg} 4510$ mouse model where the NFT formation is driven by strong overexpression of human mutated P301L tau (13-fold increase versus 2-fold increase in P301S mice; Allen et al., 2002; 
SantaCruz et al., 2005). The first NFTs in this model are detectable already at 2.5 months of age. However, the circuits in human patients are challenged by NFT formation believed to happen through interneuronal transfer of tau fibrils and subsequent seeding and aggregation. We tried to model this mechanism by injecting exogenous fibrils into circuits that normally do not develop strong fibrillar pathology-no study so far investigated the functional consequences of such NFT formation in vivo at single neuron level.

Taken together our results point to the pathological role of tau species present before formation of NFTs. The source for all these species is mislocalized tau protein present in the somatodendritic compartment, which becomes misfolded and pathologically phosphorylated. In addition, it is reasonable to assume that species could also involve small tau fibrils which still do not reach critical concentration to form NFTs. NFT formation, on the other hand, might be the part of disease 'off pathway' as proposed by Bradley Hyman and co-authors (Spires-Jones et al., 2011; Kopeikina et al., 2012; Kuchibhotla et al., 2014).

The results of this study could have implications for the choice of the right therapeutic strategy in treating patients suffering from tauopathies. Therapy approaches should be focused on eliminating toxic soluble tau species rather than trying to eradicate NFTs. Furthermore, our results advise caution in therapeutic approaches aimed at dissolving or at preventing the formation of tau aggregates.

\section{Acknowledgements}

We thank Eric Grießinger, Sonja Steinbach, Katharina Bayer, Sarah Hanselka, Michael Schmidt and Nadine Lachner for excellent technical support. We thank Dr Ilse Dewachter (Catholic University of Louvain, Belgium) for kindly providing us with tau-PFFs. We thank Dr Michel Goedert (Cambridge, UK) for kindly providing us with P301S mice. We gratefully thank Dr Carmelo Sgobio (DZNE, Germany) for helping in statistical analysis.

\section{Funding}

This work was supported by the German Center for Neurodegenerative Disease (DZNE), and the Munich Cluster for Systems Neurology SyNergy (EXC 1010).

\section{Competing interests}

The authors report no competing interests.

\section{Supplementary material}

Supplementary material is available at Brain online.

\section{References}

Allen B, Ingram E, Takao M, Smith MJ, Jakes R, Virdee K, et al. Abundant tau filaments and nonapoptotic neurodegeneration in transgenic mice expressing human P301S tau protein. J Neurosci 2002; 22: 9340-51.

Andermann ML, Kerlin AM, Reid RC. Chronic cellular imaging of mouse visual cortex during operant behavior and passive viewing [Internet]. Front Cell Neurosci 2010; 4. Available from: https:// www.ncbi.nlm.nih.gov/pmc/articles/PMC2854571/ (12 August 2018, date last accessed).

Arriagada PV, Growdon JH, Hedley-Whyte ET, Hyman BT. Neurofibrillary tangles but not senile plaques parallel duration and severity of Alzheimer's disease. Neurology 1992; 42: 631-9.

Ballatore C, Lee VM-Y, Trojanowski JQ. Tau-mediated neurodegeneration in Alzheimer's disease and related disorders. Nat Rev Neurosci 2007; 8: 663-72.

Berger Z, Roder H, Hanna A, Carlson A, Rangachari V, Yue M, et al. Accumulation of pathological tau species and memory loss in a conditional model of tauopathy. J Neurosci 2007; 27: 3650-62.

de Calignon A, Fox LM, Pitstick R, Carlson GA, Bacskai BJ, SpiresJones TL, et al. Caspase activation precedes and leads to tangles. Nature 2010; 464: 1201-4.

Clavaguera F, Bolmont T, Crowther RA, Abramowski D, Frank S, Probst A, et al. Transmission and spreading of tauopathy in transgenic mouse brain. Nat Cell Biol 2009; 11: 909-13.

Crochet S, Petersen $\mathrm{CCH}$. Correlating whisker behavior with membrane potential in barrel cortex of awake mice. Nat Neurosci 2006; 9: 608-10.

Delobel P, Lavenir I, Fraser G, Ingram E, Holzer M, Ghetti B, et al. Analysis of tau phosphorylation and truncation in a mouse model of human tauopathy. Am J Pathol 2008; 172: 123-31.

Gómez-Isla T, Hollister R, West H, Mui S, Growdon JH, Petersen RC, et al. Neuronal loss correlates with but exceeds neurofibrillary tangles in Alzheimer's disease. Ann Neurol 1997; 41: 17-24.

Guizar-Sicairos M, Thurman ST, Fienup JR. Efficient subpixel image registration algorithms. Opt Lett 2008; 33: 156-8.

Hoffmann NA, Dorostkar MM, Blumenstock S, Goedert M, Herms J. Impaired plasticity of cortical dendritic spines in P301S tau transgenic mice. Acta Neuropathol Commun 2013; 1: 1.

Holtmaat A, Bonhoeffer T, Chow DK, Chuckowree J, De Paola V, Hofer $\mathrm{SB}$, et al. Long-term, high-resolution imaging in the mouse neocortex through a chronic cranial window. Nat Protoc 2009; 4: 1128-44.

Hutton M, Lendon CL, Rizzu P, Baker M, Froelich S, Houlden H, et al. Association of missense and 5 -splice-site mutations in tau with the inherited dementia FTDP-17. Nature 1998; 393: 702-5.

Iba M, Guo JL, McBride JD, Zhang B, Trojanowski JQ, Lee VM-Y. Synthetic tau fibrils mediate transmission of neurofibrillary tangles in a transgenic mouse model of Alzheimer's-like tauopathy. J Neurosci 2013; 33: 1024-37.

Kerr JND, Denk W. Imaging in vivo: watching the brain in action. Nat Rev Neurosci 2008; 9: 195-205.

Ko H, Hofer SB, Pichler B, Buchanan KA, Sjöström PJ, Mrsic-Flogel TD. Functional specificity of local synaptic connections in neocortical networks. Nature 2011; 473: 87-91.

Kopeikina KJ, Hyman BT, Spires-Jones TL. Soluble forms of tau are toxic in Alzheimer's disease. Transl Neurosci 2012; 3: 223-33.

Kuchibhotla KV, Wegmann S, Kopeikina KJ, Hawkes J, Rudinskiy N, Andermann ML, et al. Neurofibrillary tangle-bearing neurons are functionally integrated in cortical circuits in vivo. Proc Natl Acad Sci U S A 2014; 111: 510-14.

Lee VM, Goedert M, Trojanowski JQ. Neurodegenerative tauopathies. Annu Rev Neurosci 2001; 24: 1121-59.

Marinković P, Godinho L, Misgeld T. Generation and screening of transgenic mice with neuronal labeling controlled by Thy1 regulatory elements. Cold Spring Harb Protoc 2015; 2015: 875-82. 
Menkes-Caspi N, Yamin HG, Kellner V, Spires-Jones TL, Cohen D, Stern EA. Pathological tau disrupts ongoing network activity. Neuron 2015; 85: 959-66.

Nelson PT, Alafuzoff I, Bigio EH, Bouras C, Braak H, Cairns NJ, et al. Correlation of Alzheimer disease neuropathologic changes with cognitive status: a review of the literature. J Neuropathol Exp Neurol 2012; 71: 362-81.

Peeraer E, Bottelbergs A, Van Kolen K, Stancu I -C, Vasconcelos B, Mahieu M, et al. Intracerebral injection of preformed synthetic tau fibrils initiates widespread tauopathy and neuronal loss in the brains of tau transgenic mice. Neurobiol Dis 2015; 73: 83-95.

Poorkaj P, Bird TD, Wijsman E, Nemens E, Garruto RM, Anderson L, et al. Tau is a candidate gene for chromosome 17 frontotemporal dementia. Ann Neurol 1998; 43: 815-25.

Rocher AB, Crimins JL, Amatrudo JM, Kinson MS, Todd-Brown MA, Lewis J, et al. Structural and functional changes in tau mutant mice neurons are not linked to the presence of NFTs. Exp Neurol 2010; 223: 385-93.

Rose T, Jaepel J, Hübener M, Bonhoeffer T. Cell-specific restoration of stimulus preference after monocular deprivation in the visual cortex. Science 2016; 352: 1319-22.

SantaCruz K, Lewis J, Spires T, Paulson J, Kotilinek L, Ingelsson M, et al. Tau suppression in a neurodegenerative mouse model improves memory function. Science 2005; 309: 476-81.
Schön C, Hoffmann NA, Ochs SM, Burgold S, Filser S, Steinbach S, et al. Long-term in vivo imaging of fibrillar tau in the retina of P301S transgenic mice. PLOS ONE 2012; 7: e53547.

Spillantini MG, Murrell JR, Goedert M, Farlow MR, Klug A, Ghetti B. Mutation in the tau gene in familial multiple system tauopathy with presenile dementia. Proc Natl Acad Sci U S A 1998; 95: 773741.

Spires-Jones TL, Kopeikina KJ, Koffie RM, de Calignon A, Hyman BT. Are tangles as toxic as they look? J Mol Neurosci 2011; 45: 438-44.

Stancu I-C, Vasconcelos B, Ris L, Wang P, Villers A, Peeraer E, et al. Templated misfolding of Tau by prion-like seeding along neuronal connections impairs neuronal network function and associated behavioral outcomes in Tau transgenic mice. Acta Neuropathol (Berl) 2015; 129: 875-94.

Velasco A, Fraser G, Delobel P, Ghetti B, Lavenir I, Goedert M. Detection of filamentous tau inclusions by the fluorescent Congo red derivative FSB [(trans,trans)-1-fluoro-2,5bis(3-hydroxycarbonyl-4-hydroxy)styrylbenzene]. Febs Lett 2008; 582: 901-6.

Weingarten MD, Lockwood AH, Hwo SY, Kirschner MW. A protein factor essential for microtubule assembly. Proc Natl Acad Sci U S A 1975; 72: 1858-62. 